\title{
Glande Peneano - Teoría Integral y su Relación con la Triada Disfuncional (Glande Frío, Blando o Doloroso)
}

\section{Penis Glans - Integral Theory and its Relationship with Dysfunctional Triad (Cold, Soft and Painful Glans)}

\author{
Juan Fernando Uribe Arcila ${ }^{1}$ \\ ${ }^{1}$ Servicio de Urología, Hospital Pablo Tobón Uribe, Medellín, Colombia \\ Urol Colomb 2018;27:14-24.
}

Address for correspondence Juan Fernando Uribe Arcila, MD, Servicio de Urología, Hospital Pablo Tobón Uribe, Dirección postal: Carrera 48 No 19 A 40 of 1412, Medellín, Colombia (e-mail: medicinasexual@une.net.co).

\section{Resumen \\ Palabras Clave \\ - pene \\ - erección \\ - glande frío \\ - glande doloroso \\ - glande blando \\ - disfunción eréctil}

El glande peneano forma parte de un ingenioso diseño evolutivo que convirtió el pene en una compleja maquina hidráulica bicameral para la intromisión exitosa en la vagina. El objetivo del glande es tener un extremo blando, recubierto con una piel de terminales altamente sensitivas que forma parte de un tercer cuerpo cilíndrico, que debe colaborar en la tumescencia peneana sin interferir ni en la eyección del semen, ni en la evacuación de la orina, al estar las funciones reproductivas y urinarias compartidas por el mismo órgano. Los cuerpos cavernosos y el esponjoso, incluyendo el glande, comparten anatomía y fisiología en lo referente a trabéculas, túnica albugínea y llenado de sangre, pero no son idénticos en función, por lo que conceptos como rigidez axial, distribución de receptores o venoclusión varían de una a otra cámara. Adicionalmente a su discreta colaboración en la erección, el glande es el origen del llamado reflejo eyaculatorio, que es básico en el proceso sexual humano. La disfunción de esa porción terminal, incluye tres situaciones patológicas que son el glande frío, blando o doloroso, con etiología común, relacionada con factores como trauma, disfunción eréctil o cirugías tales como prostatectomía radical o cirugía uretral, con muy pocos tratamientos específicos disponibles en la actualidad y que sin embargo deberán ir mejorando por la inusitada frecuencia de esos padecimientos.

The glans arises from a clever evolving design through which that the penis was transformed into a complex bicameral hydraulic machine for successful intervention in the vagina; aiming to maintain a highly sensitive at soft end, being part of a third cylindrical body and functioning as an aid in the tumescence with no interference with the evacuation of urine or semen, as the reproductive and urinary functions are shared by the same body. The previous asseveration implies that corpora cavernosa and spongiosum, including glans, share anatomy and physiology, trabeculation, tunica albuginea and blood filled, although not identical in function yet concepts as axial rigidity, sensory receptors distribution or venoclussion, they are different from each other and dysfunction in this terminal portion, includes three pathological situations: cold, soft or painful glans, with common etiology related to factors such as trauma, erectile dysfunction and surgeries such as radical prostatectomy or urethral surgery, with few specific treatments currently available, yet they should be improving by the unusual frequency of these conditions. received

April 11, 2017

accepted

July 10, 2017

published online

March 13, 2018
DOI https://doi.org/

$10.1055 / \mathrm{s}-0038-1637012$.

ISSN 0120-789X.

eISSN 2027-0119.
Copyright (c) 2018, Sociedad Colombiana License terms de Urología. Publicado por Thieme Revinter Publicações Ltda., Rio de Janeiro, Brazil. Todos los derechos reservados.

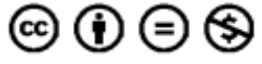




\section{Introducción}

La comunidad médica está hoy menos preocupada por los temas anatómicos, que parecieran todos entendidos a cabalidad. Sin embargo, el glande del pene es una notable excepción, puesto que la literatura disponible es exigua y dispersa, hasta el punto que el glande es inexplicablemente un capítulo casi ignorado en la erección peneana. La idea evolutiva en el pene fue crear un extraordinario sistema hidráulico para la intromisión efectiva en la cavidad vaginal, a pesar de existir una peculiar desproporción entre la rigidez de la parte dorsal del cuerpo peneano versus la ventral y su punta blanda, lo cual constituye sin embargo un diseño exitoso para lograr la mezcla adecuada entre una punta de lanza intrusiva que deposite el semen, consiga el placer y mantenga intactas las funciones evacuativas. Su integridad depende de un delicado equilibrio entre factores físicos y hemodinámicos que permiten que dicha erección sea lo suficientemente blanda en el esponjoso para una intromisión placentera, sin afectar la rigidez del cuerpo cavernoso adyacente y la capacidad de eyacular. Aunque el glande no es la clave de la erección, de su integridad depende en parte la función eréctil, puesto que en sus fallas, el paciente no estará satisfecho con los tratamientos tradicionales.

La intención de este artículo es recopilar la información obtenida por diferentes autores sobre los estudios anatómicos, fisiológicos con respecto al glande del pene, su correlación con las posibles disfunciones y un inventario de los tratamientos actualmente disponibles.

\section{Anatomía y Fisiología del Glande}

El pene se compone de tres cuerpos de tejido: un cuerpo esponjoso (CE) que termina en el glande y dos cuerpos cavernosos (CC) que funcionan como condensadores de sangre, proporcionando una armazón para lograr una erección rígida. El extremo esponjoso se expande a un glande cónico, con forma de gorro frigio, seta u hongo, que se pliega dorsal y proximal para cubrir el extremo de los cuerpos cavernosos, formando una cresta prominente, llamada corona. El cuerpo esponjoso (CE) tiene una apariencia histológica similar a la del cuerpo cavernoso (CC), puesto que ambos son tejido eréctil trabeculado, aunque con sutiles diferencias. ${ }^{1}$ Ambos cuerpos cavernosos (CC) se fusionan, con un tabique incompleto que los divide; el cuerpo esponjoso (CE), más pequeño, se encuentra en el surco ventral entre los cuerpos cavernosos (CC) y es atravesado por la uretra en la posición central $^{2,3}$ (- Fig. 1).

Mientras el glande se constituye en una punta de lanza acolchada, relativamente insensible que remata el extremo del pene, el prepucio en cambio, tiene tres zonas definidas de piel que son altamente sensitivas en conjunto. En la posición "6" del glande se encuentra una porción triangular de piel denominada frenillo o también delta peneano, un tejido conectivo muy elástico que ayuda a la fijación y a devolver como un resorte, al prepucio retraído a su posición original, siendo ricamente inervado e irrigado.

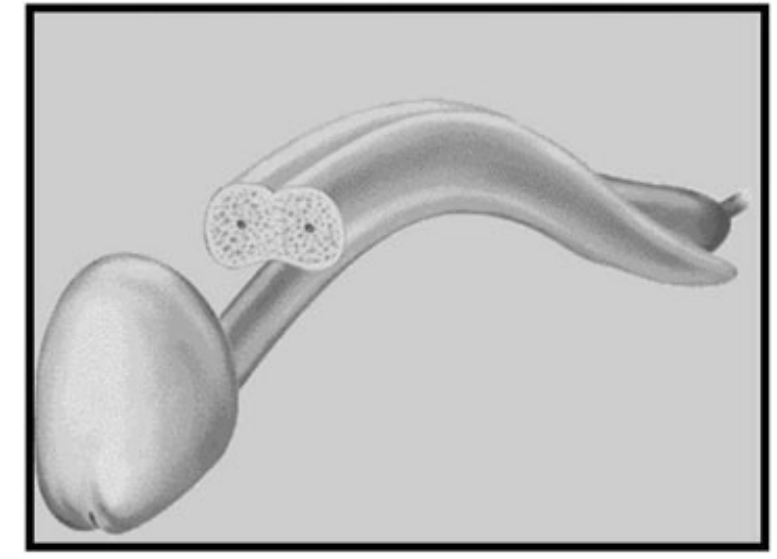

Fig. 1 El diseño tricameral con los cuerpos cavernosos (CC) seccionados y el cuerpo esponjoso (CE) intacto terminado en un glande acolchado.

Variación de 0 a 90 . Tomado de: http://www.maleinitiation.net/frenulum/philips_frenulum_questionnaire/ original_frenulum_questionnaire.html

El frenillo durante la excitación es tan sensible, que el estímulo exclusivo de esa área puede ser suficiente para desencadenar orgasmos. Ese diseño no es igual en todas las especies, puesto que existen glandes rígidos, ultradelgados, cónicos o espinosos, para ayudar en diferentes formas a la labor reproductiva. El glande puede tener diferentes grados de angulación según la longitud de ese tejido cuando actúa como resorte (Entre $0^{\circ}$ y $90^{\circ}$ ) (-Fig. 2). ${ }^{4-7}$

\section{Túnicas Peneanas y Pilares Intracavernosos}

Por debajo de las capas del prepucio (P), epitelio (E) y lámina propia (LP), el pene se encuentra cubierto por una capa de tejido conectivo y fibras musculares lisas llamada fascia superficial o dartos (DT) que es a su vez una continuación de la fascia de Colles en el periné y de la de Scarpa en el abdomen. Por debajo de ella, se encuentra una segunda capa, llamada fascia profunda penil o de Buck (PF), que es una membrana más resistente que la anterior, rodeando a ambos cuerpos cavernosos (CC) y al esponjoso (CE) de la uretra, sin cubrir el glande (GL); por último, se encuentra la túnica albugínea o vaina fibrosa (TA), la cual cumple un papel preponderante en la erección y que conforma el fibro esqueleto del pene como una estructura de múltiples capas de tejido conectivo, internas circulares y externas longitudinales, que abarcan ambos cuerpos cavernosos (CC), pero no el esponjoso (CE); en el glande (GL) las fibras elásticas están también organizadas en una capa interna circular y una capa externa longitudinal (-Fig. 3). ${ }^{8}$

La naturaleza fibrosa de la túnica albugínea, le permite al pene cierta distensión, alcanzando rápidamente su límite máximo de expansión, lo que posibilita conseguir la rigidez peneana. Los cuerpos cavernosos (CC) separados por un tabique incompleto, tienen anclajes periódicos en la capa interna circular de la túnica albugínea usando pilares intracavernosos; en el pene pendular distal por ejemplo, dichos pilares se anclan a la túnica través de los cuerpos cavernosos en las posiciones " 2 " y “ 6 " del 


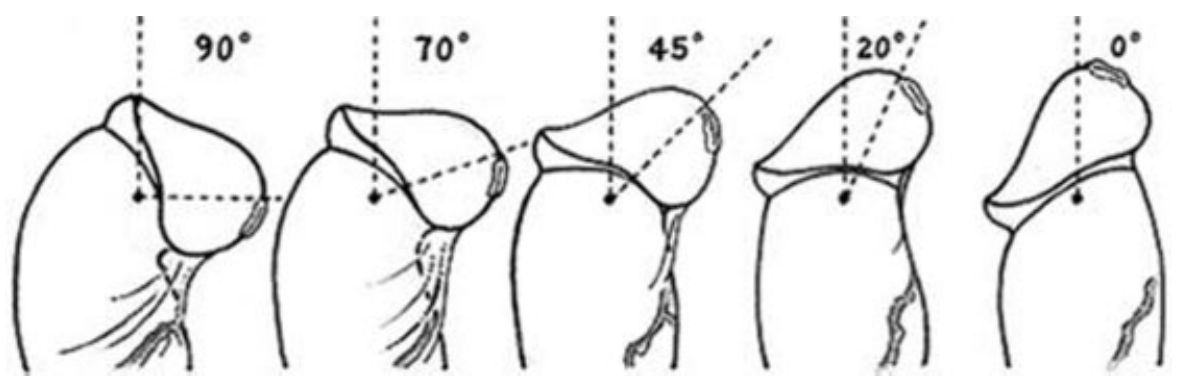

Fig. 2 Grados de angulación del glande según frenillo.

reloj, con ramificaciones menores en las posiciones “ 5 ” y “7” del reloj. La capa longitudinal es más delgada en las posiciones de las "9" y "3" del reloj, en consonancia con un mayor riesgo de fracturas peneanas en esos puntos. Lo más interesante es que ese es un diseño "anti-obstrucción"; las presiones intraespongiosas son de un tercio a la mitad de la de los cuerpos cavernosos, lo cual es una ventaja, puesto que esa presión más baja, puede prevenir la obstrucción de la uretra durante la eyaculación. Eso también puede ser la razón de la ausencia de fibras longitudinales en la posición "6" del reloj de la túnica albugínea, de tal manera que la uretra no estará nunca restringida en lo más mínimo para eyacular (-Fig. 4) ${ }^{9-19}$

\section{Irrigación y Drenaje}

La irrigación arterial del glande es simple, dependiendo casi exclusivamente de las dos arterias dorsales peneanas, que terminan formando un plexo arterial retrocoronal (-Fig. 5). Mientras el drenaje venoso está constituido por tres sistemas: El sistema superficial que permite el drenaje venoso de los tres cuerpos, comienza a nivel de pequeñas vénulas provenientes de los espacios lacunares periféricos por debajo de la túnica albugínea; esas venas forman el plexo venoso subalbugíneo que desemboca en la vena dorsal profunda; en ese sistema, las venas dorsales superficiales son pequeños canales situados en

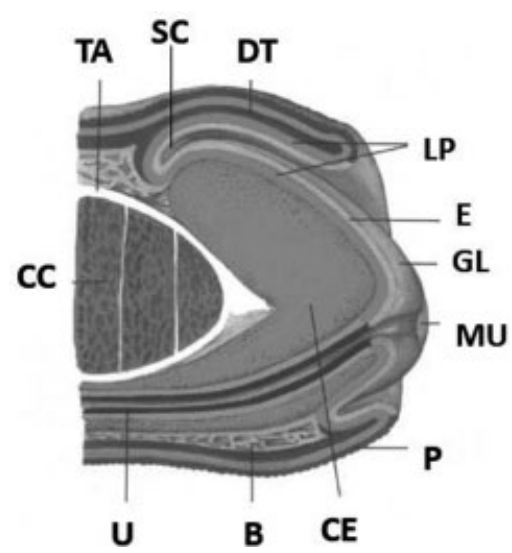

Fig. 3 Anatomía distal del pene. Abreviaciones: B, fascia penil profunda o de Buck; CC, cuerpo cavernoso; CE, cuerpo esponjoso; DT, dartos o fascia superficial; E, epitelio superficial del prepucio; GL, glande; LP, lámina propia del prepucio; MU, meato uretral; $P$, prepucio; SC, surco coronal; TA, albugínea; U, uretra. Modificado de: Stacey E. Mills (ed) (2007) Histology for Pathologists, 3rd, Lippincott, Williams and Williams, Philadelphia, chapter 38. el tejido subcutáneo del pene; es un sistema no funcional en la erección, con venas que transcurren entre ambas fascias (Colles y Buck), y desembocan en la vena dorsal superficial, la que a su vez, drena en la vena safena izquierda y menos frecuentemente en la safena derecha, en la femoral o en la epigástrica. El sistema intermedio está constituido por venas profundas a la fascia de Buck pero superficiales a la túnica albugínea; del glande emergen de 6 a 15 vénulas, que constituyen el plexo venoso retrocoronal que al confluir forman la vena dorsal profunda, recibiendo el drenaje lateral por una serie de venas llamadas circunflejas, provenientes del cuerpo esponjoso y de la uretra, lo cual es la principal vía de drenaje del glande y de los dos tercios distales del cuerpo cavernoso; las venas circunflejas se originan en el cuerpo esponjoso, pasan alrededor de los cuerpos cavernosos y se unen perpendicularmente a la vena dorsal profunda que usualmente es una sola vena, algunas veces más

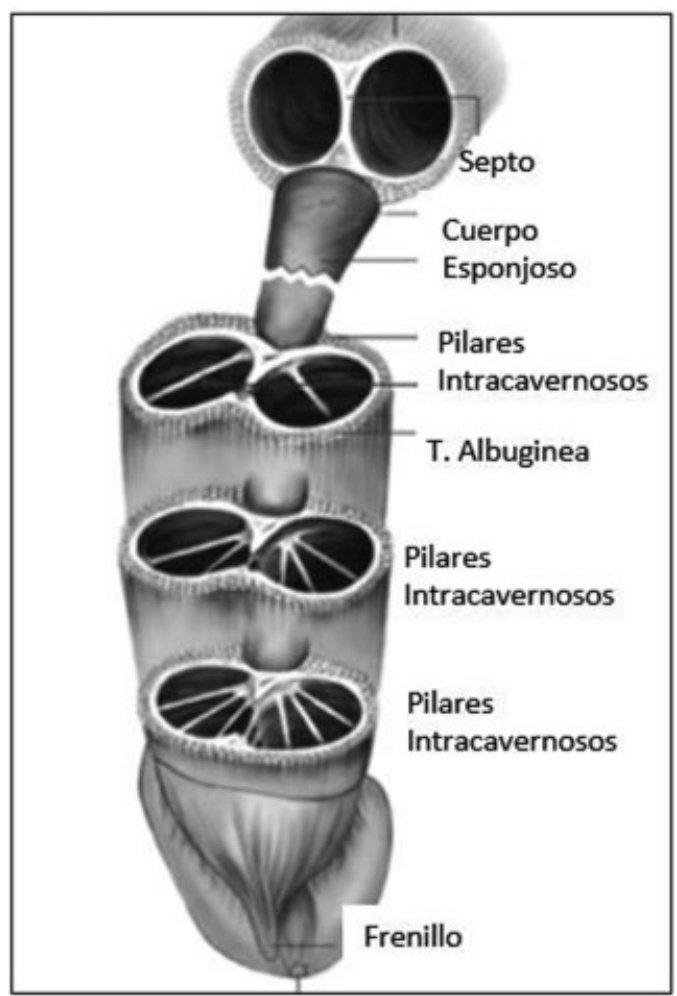

Fig. 4 Pilares intracavernosos del pene. Fonte: Hsieh $\mathrm{CH}$, Liu SP, Hsu GL. Advances inunderstanding of mammalian penile evolution, human penile anatomy and human erection physiology: clinical implications for physicians and surgeons. Med Sci Monit. 2012. 


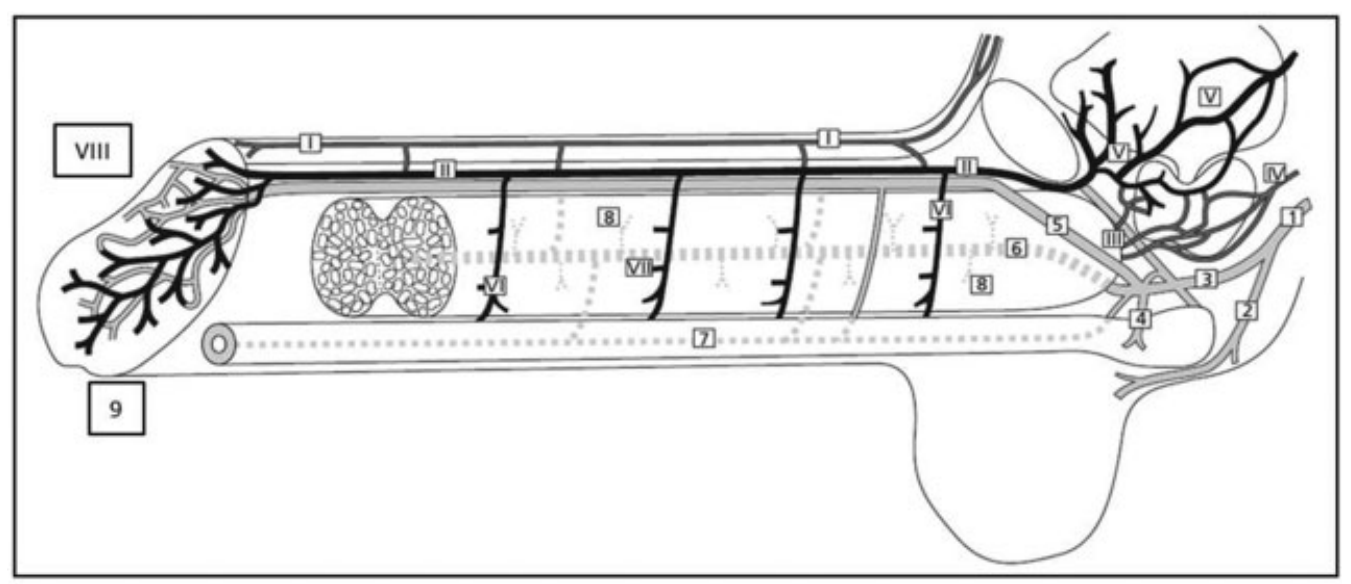

Fig. 5 Esquema vascular completo del pene. Arterias en números arábigos: 1 - Arteria pudenda interna 2- Arteria crural 3- Arteria peneana común 4- Arteria circunfleja cavernosa 5- Arterias dorsales 6- Arterias cavernosas. 7- Arteria Bulbo-Uretral. 8- Arterias helicínieas. 9- Plexo arterial retrocoronal con arteria prepucial. Venas en números romanos: I- Vena dorsal superficial II- Vena dorsal profunda III- Venas bulbares IV- Plexo pudendo V-Venas periprostáticas o Plexo de Santorini VI-Venas circunflejas VII- Venas emisarias VIII- Plexo venoso retrocoronal. Modificado de: Porst $\mathrm{H}$ and Sharlip I. "Anatomy and Physiology of Erection."

de una, por debajo de la sínfisis púbica para unirse al plexo venoso peri prostático. Ese sistema es de gran interés clínico, puesto que la fuga venosa localizada en la vena dorsal profunda, puede ser causa, bien de glande blando o de disfunción eréctil, incluso primaria en pacientes cuya hemodinámica se ve alterada en forma importante por el flujo saliente. ${ }^{20}$ Finalmente, el sistema profundo está constituido por venas que drenan los cuerpos cavernosos y esponjoso a través de las venas profundas del cuerpo cavernoso y van directo a las venas pudendas internas (- Fig. 5) ${ }^{21-23}$

\section{La Erección Glande-esponjosa}

El diseño citado anteriormente implica que la hemodinámica y la estructura del cuerpo esponjoso (CE) y el glande son por completo diferentes a la de los cuerpos cavernosos (CC). Se ha encontrado que además de colágeno, existen una gran cantidad de fibras elásticas glandulares que permiten absorber mejor las fuerzas compresivas que se generan durante la actividad sexual. Un hallazgo muy interesante es que el septo peneano parece ser flotante entre flaccidez y erección, de tal manera que en erección, dos cavidades se vuelven una sola. Sin embargo, esos estudios realizados en penes congelados de cadáveres necesitan confirmación adicional. Un pene en reposo tiene una presión de entre 0 y $5 \mathrm{~mm}$ de $\mathrm{Hg}$; durante la erección, el flujo arterial aumenta en una manera similar para los cuerpos cavernosos y el esponjoso; la presión puede alcanzar $100 \mathrm{~mm}$ $\mathrm{Hg}$ con el llenado pero hasta $220 \mathrm{~mm} \mathrm{Hg}$ con la contracción de los músculos bulbocavernosos e isquiocavernosos; sin embargo, la presión en el cuerpo esponjoso y glande es apenas de un $1 / 4$ a $1 / 2$, que la de los cuerpos cavernosos, porque la túnica albugínea, que es fina sobre el cuerpo esponjoso y prácticamente ausente sobre el glande, asegura una mínima oclusión venosa. Durante la fase de erección completa, la compresión parcial de la vena dorsal profunda y de las venas circunflejas entre la fascia de Buck y los cuerpos cavernosos congestionados contribuyen a crear la tumescencia glandular, aunque el cuerpo esponjoso y el glande funcionan más como una gran derivación arterio venosa durante esa fase. En la fase final de erección rígida, la fuerza de los músculos isquiocavernosos y bulbocavernosos, también comprime las venas y el esponjoso del pene, aumentando la congestión esponjosa y la presión en la zona del glande, fundamental para una erección cavernosa muy rígida y razonablemente rígida en el glande, que es lo normal. 2,3,24

\section{Glande y Rigidez Peneana}

La rigidez peneana se define como la habilidad del pene erecto de vencer las fuerzas axiales de la vagina durante la penetración, que se alcanza después de la relajación completa del músculo liso, una dilatación arterial máxima y la restricción efectiva del flujo venoso saliente del pene. ${ }^{25}$ Se ha sugerido que la calidad de la rigidez peneana está relacionada con cambios hemodinámicos, pero también por factores que generen en el pene las llamadas "fuerzas de bucle" (Penile buckling force factors), definidas como "la magnitud de la fuerza compresiva axial que aplicada al glande, resulta en una curvatura pronunciada de la diáfisis del pene, tal que una pequeña fuerza adicional provocaría un colapso de la estructura." ${ }^{5,26}$ Se ha propuesto que en hombres normales, la presión intracavernosa es de $90-130 \mathrm{~mm} \mathrm{Hg}$ durante la erección, pero que puede llegar a $300-400 \mathrm{~mm} \mathrm{Hg}$ cuando se adiciona la contracción de los músculos perineales; también se ha demostrado que el tercio externo y los dos tercios internos de la vagina tienen una presión de cerrado de 40 y $100 \mathrm{~mm} \mathrm{Hg}$ respectivamente, o incluso mayor según el entrenamiento muscular. ${ }^{27,28}$ La rigidez axial de la erección es determinada midiendo la resistencia del pene a doblarse o buclking, al aplicar un peso conocido sobre el glande; se debe conseguir una resistencia de 550 gramos, o una presión de $100 \mathrm{~mm} \mathrm{Hg}$, considerada la mínima para lograr la penetración vaginal. Tres factores afectan la calidad de rigidez: La presión intracavernosa, las propiedades mecánicas del tejido y la geometría del pene. En las propiedades mecánicas del tejido y geometría del pene influyen la expansibilidad cavernosa, que se traduce en la capacidad que tiene el cuerpo cavernoso (CC) a expandirse a 

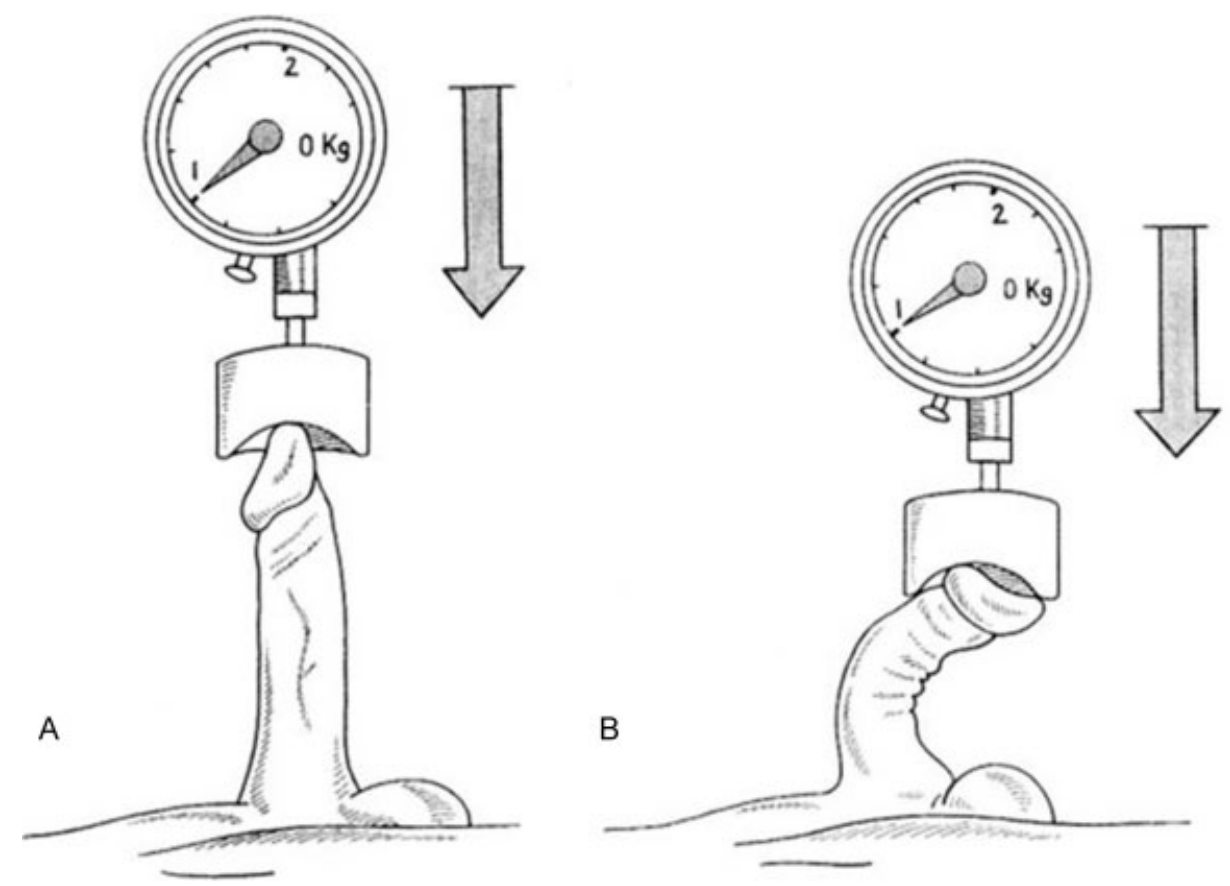

Fig. 6 Rigidez axial y resistencia a las fuerzas de torcido o de buckling, fundamentales para una erección adecuada. Tomado de: http://www. malesexualfunctionhelp.com/What-To-Expect-During-Visit.html.

un volumen máximo, con una presión intracavernosa relativamente baja; la distensibilidad de la túnica, que es la relación entre el pene totalmente erecto y el pene flácido, que mide las propiedades mecánicas de elasticidad de la túnica y finalmente la relación entre los aspectos del pene, que se refiere a la relación entre perímetro y longitud del pene flácido. La rigidez axial y no la deformación radial peneana, es entonces el parámetro físico que define mejor y objetivamente, la capacidad del pene erecto de resistirse a la deformación de las fuerzas compresivas de la vagina durante la penetración y el papel del glande es fundamental para que ese fenómeno ocurra no solo placenteramente, sino plácidamente desde el punto de vista de las fuerzas físicas y nos da una idea que el diseño no ocurre al azar (-Fig. 6). ${ }^{29-31}$

\section{Inervación del Glande}

La función del glande en la sensibilidad, es más importante que el de las otras estructuras del pene. Su piel está cubierta por un epitelio escamoso estratificado queratinizado, pero muy delgado con crestas epiteliales que varían en altura dependiendo de la región y de la edad; el epitelio sobre la superficie ventral que rodea el frenillo y la uretra es todavía más delgado que el dorsal y allí es mayor la sensibilidad. Dos tipos diferentes de bulbos genitales terminales se reconocen: Los corpúsculos nerviosos libres, firmemente superpuestos en el tope del epitelio que son pequeños y carecen de una cápsula perineural y los bulbos genitales terminales, más grandes, que están situados más profundos en la dermis y que están rodeados también por una cápsula perineural de varias capas. El glande está inervado principalmente por esas terminaciones nerviosas libres no encapsuladas, que tienen sensibilidad protopática, es decir, sensaciones primitivas, mal localizadas, incluyendo dolor, temperatura y ciertas percepciones de contacto mecánico; los terminales encapsulados más finos, son escasos y se encuentran principalmente a lo largo de la corona y el frenillo; la única parte del cuerpo con menos discriminación fina de tacto que el glande es el talón del pie. La banda estriada del prepucio masculino en la unión mucocutánea tiene una alta concentración de receptores encapsulados más sensibles; el contraste entre la sensibilidad protopática del glande y la banda estriada prepucial, rica en receptores corpusculares, es complementaria para la función erógena del pene. El glande tiene también una concentración densa de corpúsculos de Vater-Paccini, que se encuentran anclados profundamente en la dermis de la cara dorsal y de la corona, implicados en la percepción de las sensaciones vibratorias profundas, que detectan los movimientos de estiramiento y de distensión de la piel así como presiones fuertes. Los corpúsculos de Ruffini, muy sensibles al estiramiento, se observan ocasionalmente en el tejido conectivo denso de la dermis, mediante microscopía electrónica. Solo en el prepucio se encuentran células de Merkel, que son halladas frecuentemente en la piel lampiña y que tienen propiedades mecano-receptoras, haciendo que la célula libere neurotransmisores que interactúan con terminales nerviosos cercanos como un elegante mecanismo de alta sensibilidad; en el glande además de no haber terminaciones de Merkel, hay muy pocos corpúsculos de Meissner, que son altamente reactivos al tacto. La anterior información debería ser considerada en el consentimiento de la circuncisión, puesto que el prepucio es infinitamente más sensitivo que el glande (-Fig. 7). . $^{32-37}$

\section{Sensibilidad e Hipersensibilidad Peneana}

Así como la rigidez del pene está basada en el cuerpo cavernoso, la sensibilidad del pene con intención sexual, está basada en estructuras localizadas en el cuerpo esponjoso y especialmente 


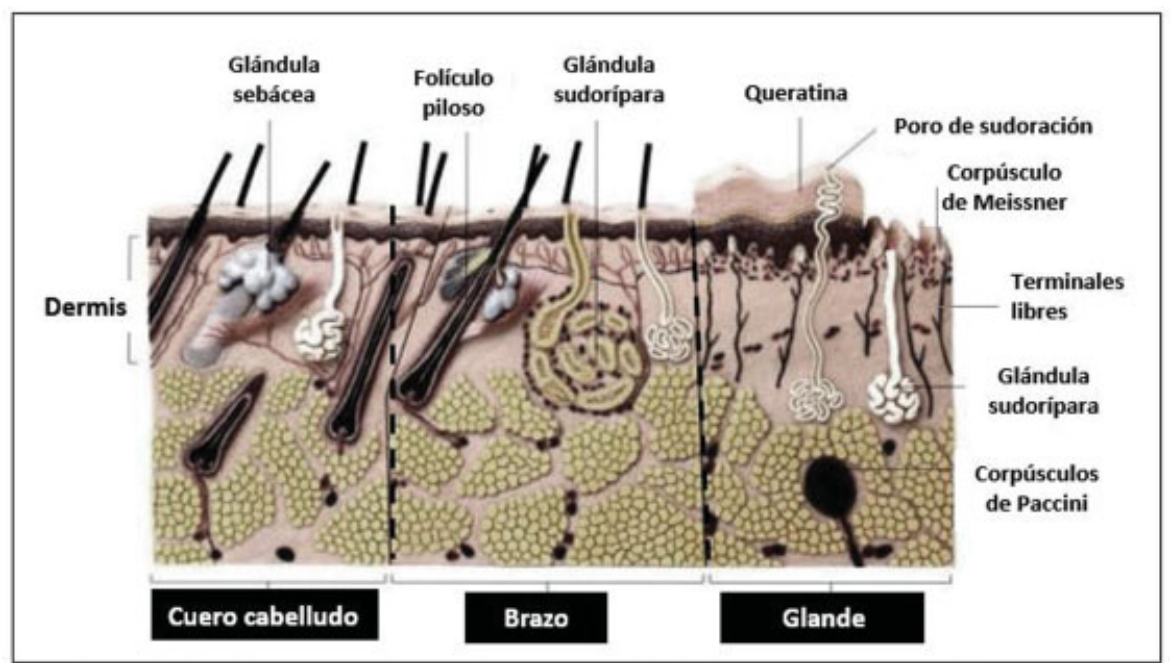

Fig. 7 Comparación de inervación superficial de la piel en tres sitios diferentes de piel. Modificado de Bear MF, Connors BW y Paradiso MA. Neurociencias. Desenredando el sistema Nervioso. Porto Alegre. 2a ed. Artmed Editora. 2002.

en el glande. Topográficamente existe una gran diferencia entre las diferentes zonas distales del pene desde el punto de vista de las aferencias que aportan al reflejo de la eyaculación, que nace en esa área con la siguiente jerarquía sensitiva: prepucio (orificio prepucial, bandas prepuciales, unión mucocutánea, prepucio externo y unión al frenillo), frenillo peneano, cuerpo del pene, meato y glande(el de menor sensibilidad). Tan rica es la inervación en la confluencia de frenillo, prepucio y surco balánico, que algunos autores hablan de un auténtico "punto G" masculino en esa zona. ${ }^{38-41}$ En patologías que impliquen compromiso neural en el pene, especialmente postquirúrgicas, los pacientes pueden presentar hiperalgesia (disociación entre la magnitud de la sensación dolorosa y el estímulo doloroso), disestesia (dificultad en localizar el área del dolor) y alodinia (dolor generado por estímulos que típicamente no serían dolorosos) (-Fig. 8).

\section{EI Reflejo Eyaculatorio}

Ciertas zonas del glande y en especial de sus envolturas son el origen del llamado Reflejo Eyaculatorio, en donde el estímulo sexual peneano viaja por un brazo sensitivo a través de la médula, llega al cerebro estimulando núcleos de recompensa y generando el proceso del orgasmo que es central y el estímulo se devuelve por un brazo motor que genera la contracción muscular lisa primero y estriada después permitiendo las dos fases de la eyaculación: la emisión y la eyección del semen (-Fig. 9).

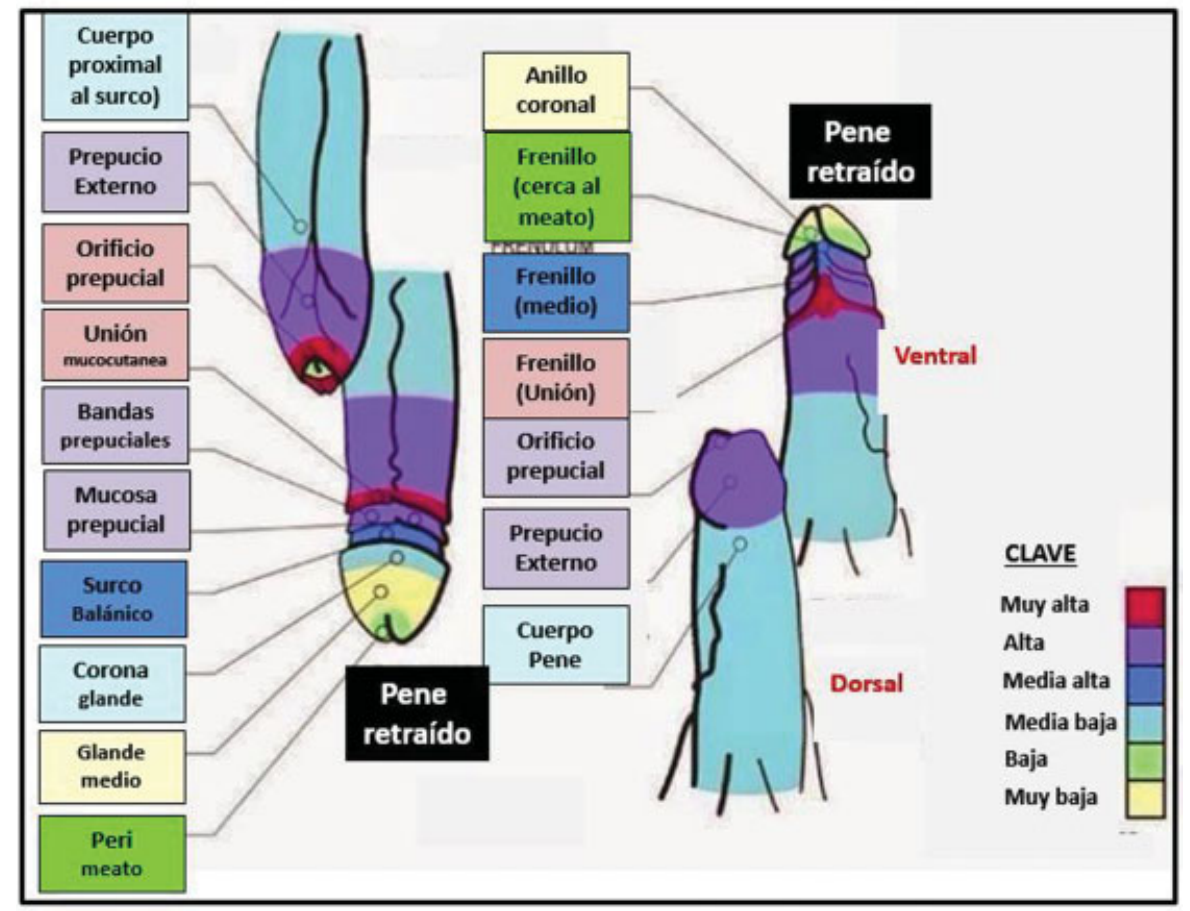

Fig. 8 Sensibilidad peneana en las zonas anatómicas. (Según Sorrells y col., 2007). ${ }^{43}$ 


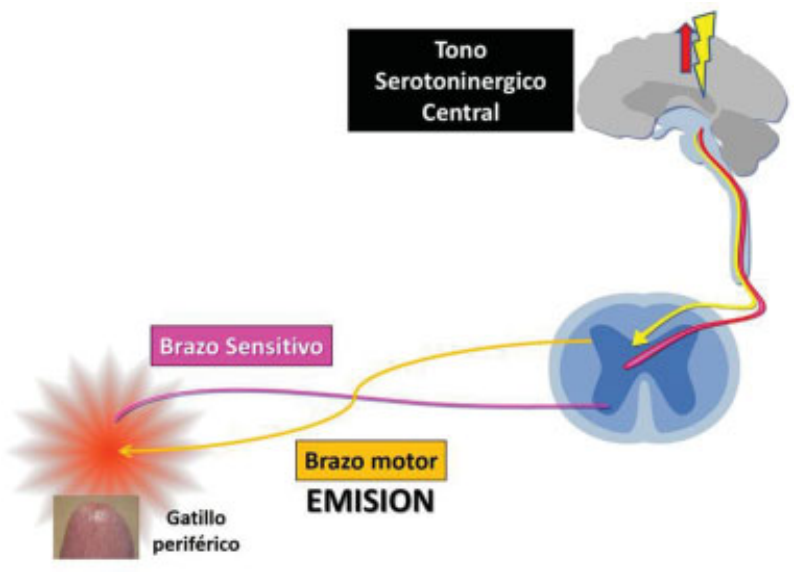

Fig. 9 Reflejo eyaculatorio. El estímulo recibido en el glande y sus envolturas viaja por un brazo sensitivo y se devuelve por un brazo motor. La devolución del estímulo está regulada por el tono serotoninergico central.

\section{Métodos Diagnósticos}

\section{Dopler Peneano}

El estudio con ultrasonido del pene es el examen más costo efectivo para la evaluación vascular y de envolturas. En el glande, uno de los aspectos a evaluar, es la presencia de comunicaciones caverno-esponjosas que normalmente se registran en casi todos los individuos y cuyo papel exacto no se ha dilucidado. Parecen ser ramificaciones de la arteria cavernosa conectándose al cuerpo esponjoso y con la red arterial uretral submucosa. Sólo unas pocas comunicaciones caverno-esponjosas se detectan en el pene flácido, mientras que de 3 a 4 se detectan en cada cuerpo cavernoso durante el inicio de la erección con el flujo dirigido hacia el cuerpo esponjoso. Usualmente no hay comunicación directa entre esos shunt caverno-esponjosos y las arterias uretrales. Cuando se alcanza la erección completa, las comunicaciones tienden a cerrarse y desaparecer con presión

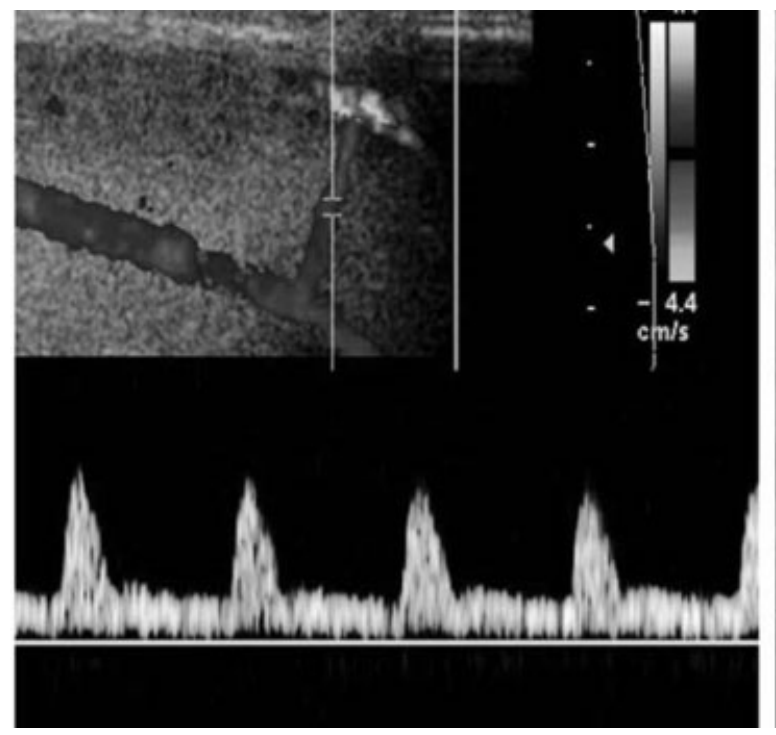

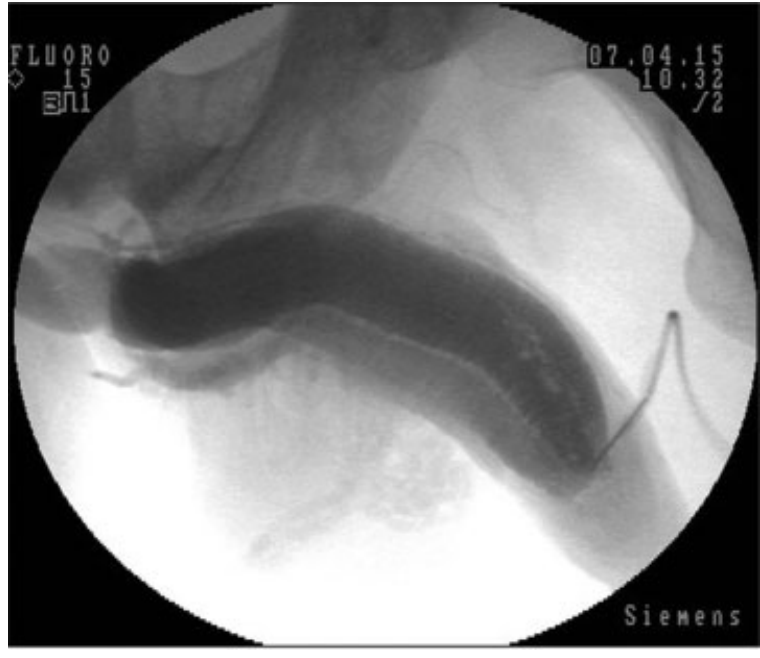

Fig. 11 Cavernosografía con espongiografía y opacificación del glande.

cercana a "0." En otros pacientes, el cierre de esas comunicaciones, no ocurre nunca durante el examen y dicha situación debe considerarse anormal ( - Fig. 10). ${ }^{42-45}$

\section{Cavernosografía con Espongiografía}

Durante una cavernosografía convencional, el glande y el cuerpo esponjoso se dibujan con el medio de contraste tanto el glande, como el cuerpo esponjoso. Sin embargo, la utilidad de ese método para patologías esponjosas es limitado. La espongiografía puede obtener información con respecto a la extensión y profundidad de zonas de fibrosis en el cuerpo esponjoso; también puede ser útil para mostrar comunicaciones caverno-esponjosas ${ }^{46}$ ( - Fig. 11).

\section{CavernotAC o CavernoRMn}

Aunque su uso no se ha extendido, el uso de métodos diagnósticos radiológicos avanzados con medio de contraste

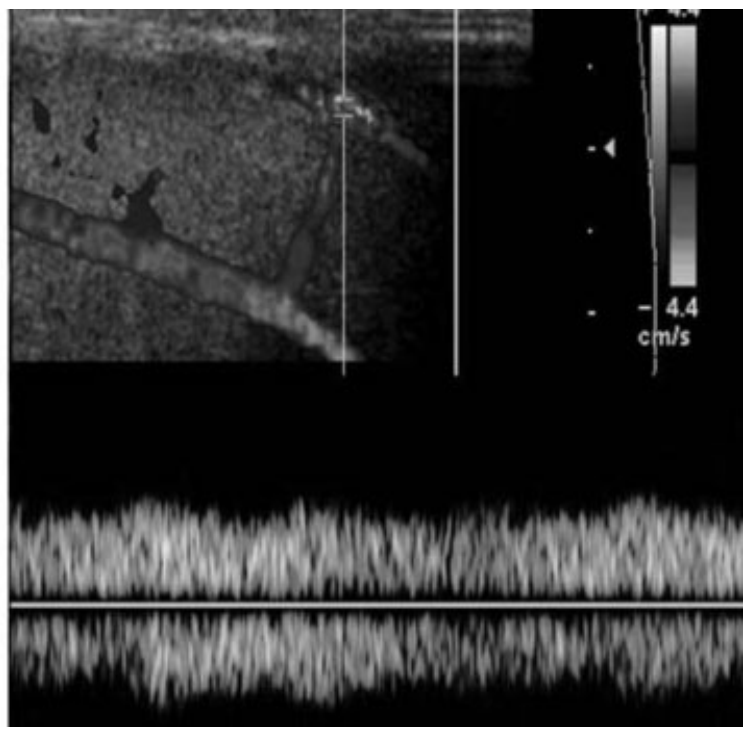

Fig. 10 Imagen dopler de las comunicaciones caverno-esponjosas. Tomada de: Bertolotto M, Lissiani A, Pizzolato R and Fute MF. "US Anatomy of the Penis: Common Findings and Anatomical Variations.” En: M. Bertolotto (Ed.). Color Doppler US of the Penis. Springer-Verlag Berlin Heidelberg 2008. 


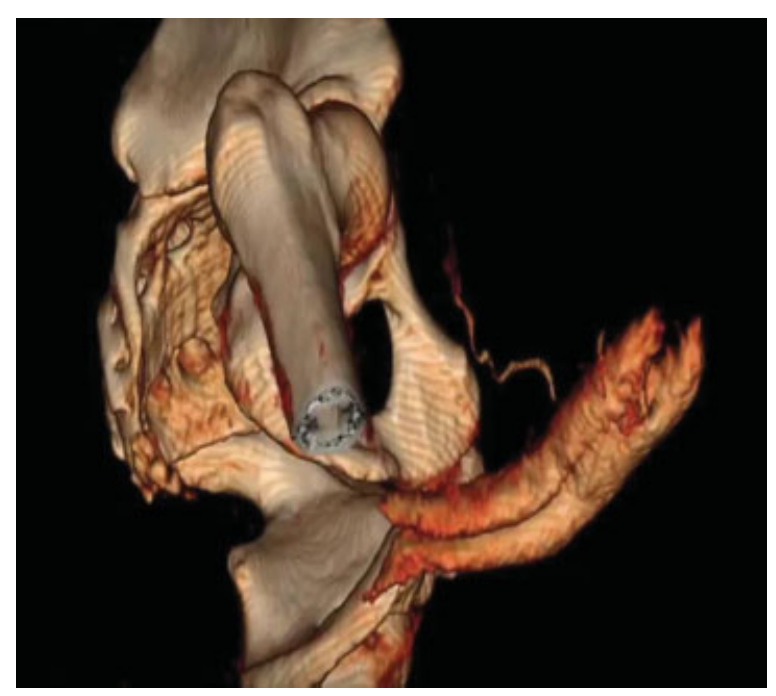

Fig. 12 Imagen de un Caverno TAC.

en el pene y reconstrucción computarizada de las imágenes suena prometedor. Pensando en el futuro, en la glande y el cuerpo esponjoso, es necesario precisar su real utilidad, puesto que además de caracterizar lesiones en el tejido, podría usarse para dibujar mejor las comunicaciones anormales y fugas venosas (-Fig. 12).

\section{Patologías del Glande}

\section{Sindrome de Glande Frio}

El glande frío o "cold glans" es una sensación de cambio permanente de temperatura en el extremo del pene que llega a ser significativa y molesta para el paciente. Aunque una causa posible es la simple pérdida de orina, en especial el goteo postmiccional, que se acumula en un prepucio estrecho desencadenando la molestia. Sin embargo, las causas más frecuentes están relacionadas con la cirugía y el trauma del cuerpo esponjoso; en ellas, una causa frecuente de frialdad, es la implantación de prótesis, que ocurre hasta en un 50\% de los pacientes; para solucionarla se ha propuesto como una solución ingeniosa, adicionar una ligadura del plexo venoso retrocoronal, la vena dorsal profunda y ramas cavernosas; aunque el efecto de esa ligadura podría considerarse paradójico. ${ }^{47}$

Una simple circuncisión, según el manejo que se haga del frenillo durante el procedimiento, puede desencadenar no solo la frialdad del glande, sino el glande doloroso o el blando tratados más adelante; por eso se recomienda prudencia en el manejo de la hemostasia en esa zona que suele ser especialmente conflictiva por la presencia de la arteria central del frenillo. Las neuropatías, en especial la diabética, pueden afectar la sensación térmica del pene, así como en otros sitios del cuerpo. También se ha reportado la sensación de glande frío, que puede volverse permanente en los pacientes que usan con frecuencia aparatos de vacío debido al mecanismo eréctil de esos aparatos por congestión venosa pasiva (cuadro 1).

\section{Sindrome de Glande Blando}

El síndrome de glande blando o "soft glans", ha llegado a ser una situación muy común en la medicina sexual. Puede
Cuadro 1 Causas de Glande Frío (Cold Glans).

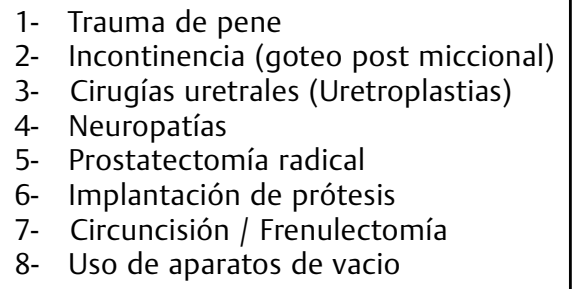

formar parte de un cuadro de disfunción eréctil estándar, en que tanto el cuerpo cavernoso (CC) como el esponjoso (CE), tienen déficit en la entrada o aumento en la salida de sangre, pero igualmente el clínico debe estar atento, puesto que es un dato semiológico muy importante en el diagnóstico de pacientes con priapismo de bajo flujo. El glande blando puede aparecer ante fugas venosas distales y ante comunicaciones caverno-esponjosas anormales, a menudo congénitas, que son un sofisticado sub diagnóstico en la disfunción eréctil. Pueden ser secuela de una cirugía previa derivativa de un priapismo, en especial cuando se usa la técnica de El Ghorab, aunque todas las derivaciones tienen riesgo. No es raro que los usuarios de aparatos de vacío relaten que no logran buena erección distal. Las otras causas conocidas son las cirugías, traumas o neuropatías que terminan siendo comunes a los tres síndromes. (cuadro 2).

\section{Sindrome de Glande Doloroso}

El síndrome de glande doloroso o "pain glans", también es una entidad relativamente frecuente, mucho más al considerar las peculiares condiciones del dolor, puesto que cada paciente vive esa experiencia de una manera diferente. El proceso de envejecimiento implica un cambio en la forma de percibir las sensaciones del pene, que de ser placenteras casi siempre, comienzan a tener tintes dolorosos en una amplia gama de grises. Prácticamente cualquier entidad inflamatoria, traumática, quirúrgica o infecciosa puede tener influencia en desarrollar puntos gatillo que influyan en la experiencia del paciente con ese delicado sensor que es el pene. (cuadro 3 ).

\section{Tratamientos}

En los tratamientos no existe un esquema dirigido exclusivamente a los problemas que afectan el glande y aunque sería deseable, la mayoría impactan primariamente

Cuadro 2 Causas de Glande Blando (Soft Glans).

\begin{tabular}{|ll|}
\hline 1- & Priapismo \\
2- & Derivaciones previas por priapismo \\
3- & Trauma de pene \\
4- & Cirugías uretrales (Uretroplastias) \\
5- & Neuropatías \\
6- & Prostatectomía radical \\
7- & Fugas venosas dorsales \\
8- & Comunicaciones caverno-esponjosas \\
9- & Uso de aparatos de vacío \\
10- & Implantación de Prótesis de pene \\
\hline
\end{tabular}


Cuadro 3 Causas de Glande Doloroso (Painful Glans).

1- Envejecimiento

2- Enfermedad de Peyronie

3- Cavernosopatía traumática crónica

4- Enfermedad de Mondor

5- Trauma de pene

6- Cirugías uretrales (Uretroplastias)

7- Neuropatías

8- Estrechez de uretra / Meato

9- Meatitis

10- Balanitis

11- Condilomatosis uretral

12- Frenillo corto

13- Circuncisión

14- Implante de cuerpos extraños

en los cuerpos cavernosos y apenas secundariamente en glande y esponjoso.

\section{Tratamiento Oral}

Algunos pacientes pueden tener éxito para mejorar el llenamiento del glande con el uso de terapias comúnmente aceptadas para la disfunción eréctil, como IPDE-5 que aumentan el flujo arterial entrante al pene. Eso se aplica tanto para el glande blando, como para el glande frío. Sin embargo, algunos de los mecanismos que causan la alteración distal, pueden no responder adecuadamente al mejor llenamiento cavernoso.

\section{Tratamiento Intrauretral}

El único medicamento que se ha postulado como una solución específica al glande blando en pacientes post radical de próstata o post implantación de prótesis de pene es el alprostadil transuretral con nombre comercial MUSE ${ }^{\circledR}$ (Medicated Urethral System for Erection) sin embargo, una combinación de factores como costo, dosis necesaria y efectos reales, limitan su uso ampliado. ${ }^{48}$

\section{Tratamiento Vasoactivo Intracavernoso}

Aunque las inyecciones intracavernosas no son un tratamiento específico, la calidad de la erección en general es muy buena. Sin embargo, los pacientes con glande blando pueden experimentar erecciones con una gran rigidez cavernosa y nula en glande y esponjoso, muy parecidas a las que se observan en casos de priapismo de bajo flujo que generan inconformidad.

\section{Inyección de Plasma Rico en Plaquetas}

El debut del PRP es la primera etapa de una serie de productos que pueden inyectarse en el pene para conseguir diferentes objetivos. Las plaquetas contienen varios factores de crecimiento tales como el plateletderived growth factor, el insulin-like growth factor-I y vascular endothelial growth factor. La idea con esa terapia, es llevar una alta concentración de factores de crecimiento a un área patológica; luego esos factores de crecimiento actúan localmente para reclutar células indiferenciadas hacia el sitio de la injuria, aumentar las mitosis en esas células e inducir neo angiogénesis. ${ }^{49,50}$

\section{Ondas de Choque al Esponjoso}

Las ondas de choque son otro tratamiento en desarrollo que se ha sugerido y que podría renovar el tejido esponjoso por diferentes mecanismos: incremento de células endoteliales, músculo liso, sintasas de $\mathrm{ON}$ y radio colágeno I/III. ${ }^{24}$

\section{Tratamientos Quirúrgicos}

Algunas cirugías pueden ser realizadas en casos especiales. En los pacientes con fuga dorsal profunda demostrada por ultrasonido y/o Cavernosografía. Las ligaduras selectivas pueden ser una buena solución, como por ejemplo la ligadura selectiva de comunicaciones caverno-esponjosas o el cierre de fístulas realizadas previamente como tratamiento de casos de priapismo de bajo flujo. Esas medidas buscan corregir las comunicaciones anormales que pueden causar glande blando. Finalmente el uso de prótesis en los casos más severos, puede corregir cualquier debilidad del pene, por la disfunción cavernosa tradicional o en casos específicos de glande blando.

\section{Tratamiento Para el Dolor}

Con respecto al tratamiento del glande doloroso, es necesario establecer la etiología. No es lo mismo tener dolor localizado por un piercing que debería ser retirado a un frenillo corto que necesita una cirugía correctora. En los casos de fibrosis, especialmente el Peyronie agudo con un compromiso de túnica albugínea cercano al glande, el paciente podría beneficiarse del uso de colchicina, que es parte del esquema tradicional de tratamiento del Peyronie. Un caso específico lo constituyen los neuromas que son proliferaciones de células de Schwann $\mathrm{u}$ otros componentes del tejido nervioso. Los neuromas pueden ser categorizados como traumáticos, de amputación, o encapsulados; el tratamiento propuesto es la resección selectiva de los nódulos. ${ }^{51}$

\section{Conclusiones}

El glande es un extremo blando y acolchado que cumple dos funciones durante la penetración del pene erecto: Ser una punta de lanza flexible y no interferir en la salida del semen que se desplaza por un cuerpo esponjoso que está rodeado por unos congestionados y rígidos cuerpos cavernosos. El glande en sí mismo es de muy baja sensibilidad, pero el frenillo y ciertas zonas del prepucio son las de mayor sensibilidad peneana y son la clave del placer sexual y el origen del reflejo eyaculatorio. Los factores que confluyen para causar su disfunción se resumen en tres situaciones y sus mezclas: Glande frío, blando y doloroso. Sin embargo, los tratamientos específicos disponibles hoy, aunque escasos e insuficientes, varían entre los vasodilatadores (orales o intracavernosos), la inyección de substancias como células madre o PRP, intrauretrales como el MUSE y las diferentes cirugías (venosa y prótesis).

Fuente de Financiación

Ninguna.

Conflicto de Intereses

Ninguno. 


\section{Agradecimientos}

Dr. Geng-Long Hzu, fuente de inspiración para esta revisión.

\section{Bibliografia}

1 Shetty SD, Farah RN. "Anatomy of erectile function" in Textbook of Erectile Dysfunction. Carson CC, Kirby R, Goldstein I, eds. Oxford: ISIS Medical Media; 1999:25-30

2 Ottenhof SR, de Graaf P, Soeterik TF, et al. Architecture of the Corpus Spongiosum: An Anatomical Study. J Urol 2016;196(03): 919-925. Doi: 10.1016/j.juro.2016.03.136

3 Hsu GL, Brock G, von Heyden B, Nunes L, Lue TF, Tanagho EA. The distribution of elastic fibrous elements within the human penis. Br J Urol 1994;73(05):566-571

4 Andersson K-E, Wagner G. Physiology of penile erection. Physiol Rev 1995;75(01):191-236

5 Udelson D, Nehra A, Hatzichristou DG, et al. Engineering analysis of penile hemodynamic and structural-dynamic relationships: Part I-Clinical implications of penile tissue mechanical properties. Int J Impot Res 1998;10(01):15-24

6 Chung B, Sommer G, Brooks J. "Anatomy of the Lower Urinary Tract and Male Genitalia". En Cambells Walsh Urology. 10a ed. Philadelphia: Elsevier-Saunders; 2012:64-66

7 Beckett SD, Purohit RC, Reynolds TM. The corpus spongiosum penis pressure and external penile muscle activity in the goat during coitus. Biol Reprod 1975;12(02):289-292

8 Andrade F, Cardoso GP, Bastos AL, Costa W, Chagas M, Babinski M. Structural and stereological analysis of elastic fibers in the glans penis of young men. Rom J Morphol Embryol 2012;53(02):393-396

9 Brock G, Hsu G-L, Nunes L, von Heyden B, Lue TF. The anatomy of the tunica albuginea in the normal penis and Peyronie's disease. J Urol 1997;157(01):276-281

10 Nitahara KS, Lue TF. "Microscopic anatomy of the penis" in Textbook of Erectile Dysfunction. Carson CC, Kirby R, Goldstein I, eds, Oxford: ISIS Medical Media; 1999:31-42

11 Hsu G-L, Brock GB, Martinez-Piñeiro L, Nunes L, Von Heyden B, Lue TF. The three-dimensional structure of the tunica albuginea: anatomical and structural levels. Int J Impot Res 1992;4:117-132

12 Goldstein AMP, Padma-Nathan H. The microarchitecture of the intracavernosal smooth muscle and the cavernosal fibrous skeleton. J Urol 1990;144(05):1144-1146

13 Goldstein AMB, Meehan JP, Zakhary R, Buckley PA, Rogers FA. New observations on microarchitecture of corpora cavernosa in man and possible relationship to mechanism of erection. Urology 1982;20(03):259-266

14 Hsu GL, Lin CW, Hsieh CH, et al. Distal ligament in human glans: a comparative study of penile architecture. J Androl 2005;26(05): 624-628

15 Hsu GL, Hsieh CH, Wen HS, et al. Anatomy of the human penis: the relationship of the architecture between skeletal and smooth muscles. J Androl 2004;25(03):426-431

16 Humphrey PA. “Genital Skin and Urethral Anatomy". Tomado de: Current Clinical Urology: Urethral Reconstructive Surgery Edited by: S.B. Brandes, Totowa, NJ: Humana Press; 2008

17 Hsieh CH, Liu SP, Hsu GL, et al. Advances in understanding of mammalian penile evolution, human penile anatomy and human erection physiology: clinical implications for physicians and surgeons. Med Sci Monit 2012;18(07):RA118-RA125

18 Mundy AR, Healy JC. Muscles and fasciae of the perineum: true pelvis, pelvic floor and perineum. In: Standring S. ed. Gray's Anatomy. 39th ed. London: Elsevier Churchill Livingstone; 2005: 1365-1371

19 Hsu GL. Hypothesis of human penile anatomy, erection hemodynamics and their clinical applications. Asian J Androl 2006;8(02):225-234
20 Uribe JF. Fuga venosa dorsal exclusiva como causa de disfunción eréctil primaria o no estándar. Vídeo ganador en el Congreso Nacional de Urología de Cali en 2013. Disponible en: https:// www.youtube.com/watch?v=eXzr3q9T8Lw

21 Porst H, Sharlip I. "Anatomy and Physiology of Erection. Chapter 3". En: Porst H and Buvat J. Standard Practice in Sexual Medicine. Malden, Massachusetts: Blackwell Publishing; 2006

22 Juskiewenski S, Vaysse PH, Moscovici J, et al. A study of the arterial blood supply of the penis. Anat Clin 1982;4:101-107

23 Wagner G, Willis EA, Bro-Rasmussen F, Nielsen MH. New theory on the mechanism of erection involving hitherto undescribed vessels. Lancet 1982;1(8269):416-418

24 Reed-Maldonado AB, Lue TF. Learning Penile Anatomy to Improve Function. J Urol 2016;196(03):643-644

25 Erdoğru T, Savaş M, Yilmaz N, Baykara M. Are normal hemodynamic responses invariably associated with normal penile rigidity and potency? Int J Impot Res 2001;13(01):10-13, discussion 14-15

26 Udelson D, Park K, Sadeghi-Nejad H, Salimpour P, Krane RJ, Goldstein I. Axial penile buckling forces vs Rigiscan radial rigidity as a function of intracavernosal pressure: why Rigiscan does not predict functional erections in individual patients. Int J Impot Res 1999;11(06):327-337, 337-339

27 Levine LA, Lenting EL. Use of nocturnal penile tumescence and rigidity in the evaluation of male erectile dysfunction. Urol Clin North Am 1995;22(04):775-788

28 Lavoisier P, Aloui R, Iwaz J, Kokkidis MJ. [The physiology of penile rigidity]. Prog Urol 1992;2(01, Suppl 2):119-127

29 García GM, Mendoza LA. "Medidor de la presión axial del pene. Tesis de grado". Escuela de Ingenieros de Antioquia. Facultad de Ingeniería biomédica, Convenio CES-EIA, 2002

30 Basar MM, Atan A, Tekdogan UY. New concept parameters of RigiScan in differentiation of vascular erectile dysfunction: is it a useful test? Int J Urol 2001;8(12):686-691

31 Frohrib DA, Goldstein I, Payton TR, Padma-Nathan H, Krane RJ. Characterization of penile erectile states using external computerbased monitoring. J Biomech Eng 1987;109(02):110-114

32 Cold CJ, Taylor JR. The prepuce. BJU Int 1999;83(Suppl 1):34-44

33 Halata Z, Munger BL. The neuroanatomical basis for the protopathic sensibility of the human glans penis. Brain Res 1986;371(02):205-230

34 Halata Z, Spaethe A. Sensory innervation of the human penis. Adv Exp Med Biol 1997;424:265-266

35 Gould VE, Moll R, Moll I, Lee I, Franke WW. Neuroendocrine (Merkel) cells of the skin: hyperplasias, dysplasias, and neoplasms. Lab Invest 1985;52(04):334-353

36 Winkelmann RK. The mucocutaneous end-organ; the primary organized sensory ending in human skin. AMA Arch Derm 1957; 76(02):225-235

37 Xia JD, Han YF, Pan F, Zhou LH, Chen Y, Dai YT. Clinical characteristics and penile afferent neuronal function in patients with primary delayed ejaculation. Andrology 2013;1(05):787-792

38 Alwaal A, Breyer BN, Lue TF. Normal male sexual function: emphasis on orgasm and ejaculation. Fertil Steril 2015;104(05): 1051-1060

39 Holstege G, Georgiadis JR, Paans AM, Meiners LC, van der Graaf FH, Reinders AA. Brain activation during human male ejaculation. J Neurosci 2003;23(27):9185-9193

40 Perry JF. Do men have a G-spot? Aust For 1988;2:37-41

41 Sorrells ML, Snyder JL, Reiss MD, et al. Fine-touch pressure thresholds in the adult penis. BJU Int 2007;99(04):864-869

42 Bertolotto M, Neumaier CE, Martinoli C, Quaia E, Savoca G. Color Doppler appearance of penile cavernosal-spongiosal communications in patients with normal and impaired erection. Eur Radiol 2002;12(09):2287-2293

43 Droupy S, Giuliano F, Jardin A, Benôit G. Cavernospongious shunts: anatomical study of intrapenile vascular pathways. Eur Urol 1999;36(02):123-128 
44 Gotta SF, Kassaniou S, Kokoua A, Gnanazan NG. [Anatomic study of arterial and venous cavernospongious shunt in the human penis: surgical findings]. Ann Urol (Paris) 2003;37(03): 135-139

45 Bertolotto M, Lissiani A, Pizzolato R, Fute MF. "US Anatomy of the Penis: Common Findings and Anatomical Variations". En: M. Bertolotto (Ed.). Color Doppler US of the Penis. Springer-Verlag Berlin Heidelberg; 2008

46 Juventino VG, Ernesto NS, Francisco GT. Espongiografía: utilidad en el diagnóstico integral de la estenosis de uretra masculina. Bol Col MX Urol 1994;11(02):105-110

47 Hsu GL, Hill JW, Hsieh CH, Liu SP, Hsu CY. Venous ligation: a novel strategy for glans enhancement in penile prosthesis implantation. BioMed Res Int 2014;2014:923171
48 Chew KK, Stuckey BG. Use of transurethral alprostadil (MUSE) (prostaglandin E1) for glans tumescence in a patient with penile prosthesis. Int J Impot Res 2000;12(03):195-196

49 Runels C, Melnick H, Debourbon E, et al. A pilot study of the effect of localized injections of autologous platelet rich plasma (PRP) for the treatment of female sexual dysfunction. J Womens Health Care 2014;3:1000169

50 Farmer M, Yoon H, Goldstein I. Future Targets for Female Sexual Dysfunction. J Sex Med 2016;13(08):1147-1165. Doi: 10.1016/j. jsxm.2016.05.016

51 Park HJ, Kim TN, Baek SR, Lee KM, Choi KU, Park NC. Penile Traumatic Neuroma: A Late Complication of Penile Dorsal Neurotomy to Treat Premature Ejaculation. Sex Med 2016;4 (03):e221-e224. Doi: 10.1016/j.esxm.2016.04.003 